Published in final edited form as:

Am J Ophthalmol. 2005 December ; 140(6): 1014-1019.

\title{
Imaging Polarimetry in Central Serous Chorioretinopathy
}

\author{
MASAHIRO MIURA, MD, ANN E. ELSNER, PhD, ANKE WEBER, MD, MICHAEL C. CHENEY, \\ MS, MASAHIRO OSAKO, MD, MASAHIKO USUI, MD, and TAKUYA IWASAKI, MD \\ From the Department of Ophthalmology, Tokyo Medical University, Tokyo, Japan (M.M., M.O., M.U., \\ T.I.); and The Schepens Eye Research Institute and Harvard Medical School, Boston, \\ Massachusetts (A.E.E., A.W., M.C.C.).
}

\section{Abstract}

PURPOSE-To evaluate a noninvasive technique to detect the leakage point of central serous chorioretinopathy (CSR), using a polarimetry method.

DESIGN—Prospective cohort study.

SETTING: Institutional practice.

PATIENTS: We examined 30 eyes of 30 patients with CSR.

MAIN OUTCOME MEASURES: Polarimetry images were recorded using the GDx-N (Laser Diagnostic Technologies). We computed four images that differed in their polarization content: a depolarized light image, an average reflectance image, a parallel polarized light image, and a birefringence image. Each polarimetry image was compared with abnormalities seen on fluorescein angiography.

RESULTS-In all eyes, leakage area could be clearly visualized as a bright area in the depolarized light images. Michelson contrasts for the leakage areas were $0.58 \pm 0.28$ in the depolarized light images, $0.17 \pm 0.11$ in the average reflectance images, $0.09 \pm 0.09$ in the parallel polarized light images, and $0.11 \pm 0.21$ in the birefringence images from the same raw data. Michelson contrasts in depolarized light images were significantly higher than for the other three images $(P<.0001$, for all tests, paired $t$ test). The fluid accumulated in the retina was well-visualized in the average and parallel polarized light images.

CONCLUSIONS-Polarization-sensitive imaging could readily localize the leakage point and area of fluid in CSR. This may assist with the rapid, noninvasive assessment of CSR.

CENTRAL SERous chorioretinopathy (CSR) IS a common disorder that typically affects middle-aged adults, predominantly male, and is characterized by serous retinal detachment with leakage at the level of retinal pigment epithelium. ${ }^{1}$ The serous retinal detachment in CSR resolves spontaneously in most of the cases. ${ }^{1}$ In some cases, however, the resolution of the serous retinal detachments is delayed and patients may develop permanent visual loss. ${ }^{1,2}$ For these cases, laser photocoagulation at the leakage point may be recommended if the leakage points are located outside the fovea. ${ }^{1}$ Localization of the leakage point is crucial for the treatment of CSR as typically ascertained in fluorescein angiography. Despite the widespread acceptance of fluorescein angiography, its application has been restricted because of the possibility of severe complication and the discomfort to patients, ${ }^{3}$ as well as the time needed to perform the test. Thus, it is important to evaluate the potentiality of other techniques.

Inquiries to Masahiro Miura, Department of Ophthalmology, Tokyo Medical University, Kasumigaura Hospital, 3-20-1 Chuo, Ami, Inashiki, Ibaraki 300-0395 Japan; fax +81-298-87-7656; e-mail: m-miura@ tokyomed.ac.jp

Supported in part by NIH NEI EYO7624 grant to Ann E. Elsner, PhD. 
Recently, a polarimetry technique was developed to selectively emphasize the different layers of the retina. ${ }^{4-6}$ In the original conceptualization, the thickness of the retinal nerve fiber layer is estimated using the birefringent component of light returning from the ocular fundus, ${ }^{4}$ since the light from the superficial structures retains a high degree of polarization. Building upon this concept, images of the superficial structures of the fundus can be enhanced by using light that is polarization preserving. In contrast, images of deep retinal lesions can be obtained using the depolarized light remaining after the removal of the polarization preserving light. 5, 6 Depolarized light images show enhanced contrast of drusen 6 and hyperpigmentation, ${ }^{7}$ and visualize retinal vessels as dark, high contrast structures that block the light from reaching and returning from the deeper layers. 5,8 The morphologic changes associated with CSR that occur at the leakage area in the deeper layers seen on histology, ${ }^{1}$ might induce the multiple scattering of the light, which would be detected in the depolarized light images. ${ }^{9}$ Furthermore, the serous retinal detachment can be visualized on optical coherence tomography (OCT) 10,11 and, in some cases, also the focal leakage area or changes to the retinal pigment epithelium, such as fibrinous exudates, are seen. We hypothesized that the polarimetry technique is useful to detect the leakage area in CSR without the injection of fluorescein dye. Additionally, the images may clearly denote the area of leakage and other features including fundus changes, occurring potentially in either acute or chronic CSR. In this study, we analyzed CSR with imaging polarimetry, which provides a method for the noninvasive assessment of CSR.

\section{SUBJECTS AND METHODS}

WE PROSPECTIVELY EXAMINED 30 EYES OF 30 JAPANESE patients, 26 males and four females, 30 to 64 years (mean: 45.9 years), with CSR. Durations of the symptoms of CSR ranged from 1 week to 6 months (mean 5.7 weeks). Before testing, all patients received a detailed explanation of the study procedure and signed consent forms approved by the Institutional Review Board of Tokyo Medical University. The leakage area could be clearly localized in fluorescein angiography images in all eyes. The OCT images (Humphrey model 2000; Humphrey Instruments, San Leandro, California) showed presence of serous retinal detachment in all eyes. In the OCT images, a small area of retinal pigment epithelial detachment, corresponding to the area of dye leakage, was observed in 11 eyes, and a highly reflective lesion bridging the detached neurosensory retina and the retinal pigment epithelium was observed in one eye.

\section{POLARIMETRY IMAGES}

We used data from the GDx-N (Laser Diagnostic Technologies, San Diego, California). This instrument is a confocal scanning laser ophthalmoscope that is widely used for estimating the thickness of the retinal nerve fiber layer in the glaucoma clinic. ${ }^{12}$ Linearly polarized light at $780 \mathrm{~nm}$ scans the retina in a raster pattern, 15 by 15 degree visual angle. To minimize the corneal birefringence, the GDx-N uses a fixed compensator with a magnitude of $60 \mathrm{~nm}$ and a polarization axis of 15 degree nasally downward (single-pass retardance), which is uniform across the pupil plane. The returning light is separated into two beams by a polarizing beam splitter: light of the same polarization as the illumination light, digitized at the parallel polarized detector, and light polarized at 90 degree to the illumination light, digitized at the crossed detector. There are 20 image pairs that vary systematically in illumination polarization angle, and each image in the series is saved as $256 \times 256$ pixels with 8 bits of grayscale. After correction for eye movement, a depolarized light image was computed from the portion of the light that was unmodulated for each pixel, for the crossed detector. ${ }^{5-8}$ The following images in order from least to most polarization content were also computed: (1) an average reflectance image, as the grand mean of the light returning to both detectors for all input polarization states, this image is similar to that obtained with a typical confocal scanning laser ophthalmoscope ophthalmoscope $^{6}$; (2) a parallel polarized light image, as the average intensity for the parallel 
polarized light minus the depolarized light contribution; and (3) a birefringence image, from the modulation of the light returning to the crossed detector.

Intensities of the computed polarimetry images varied widely, and as expected the depolarized light images and birefringence images were too dark for subjective evaluation without further manipulation. For better visualization, the distribution of intensities in each image was adjusted as follows: minimum to maximum intensities in the images were converted to a grayscale that ranged from 0 to 255 grayscale units (Adobe Photoshop, San Jose, California).

For the objective evaluation of the visibility of leakage area in polarimetry images, we calculated the contrast without any adjustment of intensities. We selected one leakage area from each eye. The average intensity of a $4 \times 4$ pixels region of retina for the leakage area and the average intensity of the same size region adjacent to this area were both calculated. Michelson contrast was calculated for objective evaluation as follows:

Michelson contrast $=\left(\mathrm{I}_{\text {leakage }}-\mathrm{I}_{\text {adjacent }}\right) /\left(\mathrm{I}_{\text {leakage }}+\mathrm{I}_{\text {adjacent }}\right)$ where $\mathrm{I}_{\text {leakage }}$ was the average grayscale value on the leakage area and $\mathrm{I}_{\text {adjacent }}$ was the average grayscale value adjacent to the leakage area.

\section{RESULTS}

in The Depolarized light images, the Leakage areas could be clearly visualized as bright areas in all eyes (Figures 1, 2, 3, and 4). These bright areas were single bright dots in three eyes (Figures 1 and 2), and a single bright dot accompanied by multiple bright dots in 27 eyes (Figures 3, and 4). However, in all eyes the leakage area could hardly be detected in the parallel polarized images, the average reflectance images, or the birefringence images. The neurosensory retinal detachment was clearly localized as a large, dark area in the parallel polarized and average reflectance images, of similar or somewhat greater size compared with that seen in the depolarized light images, which emphasized the deeper layers. A bow-tie appearance in the birefringence image was served in 29 eyes (Figures 1, 3, and 4), when present indicating the location of the fovea.

The cases were separated into groups according to the findings of OCT at the leakage area. Of the 11 eyes that visualized a pigment epithelial detachment, two eyes showed a single bright dot in the depolarized light image(Figure 1), whereas nine eyes showed a single bright dot accompanied by multiple bright dots. In the eye with the bridged lesion in OCT, the leakage area showed a single bright dot surrounded by a high intensity area (Figure 2). In the eyes without specific findings at the leakage area in OCT, the leakage area showed a single bright dot accompanied by multiple bright dots in all eyes in the depolarized images (Figures 3 and 4).

In $20(67 \%)$ of the 30 eyes, leakage areas were well defined in the depolarized light images (Figures 1,2, and 3), and showed sufficient quality for the guiding focal photocoagulation. In other 10 eyes (33\%), leakage areas were surrounded by comparatively large bright spots (Figure 4 ), or relatively blurred. In these eyes, detection of the areas for focal photocoagulation was difficult in the depolarized light images.

For all cases, Michelson contrasts of the leakage area were $0.58 \pm 0.28$ (depolarized light), $0.17 \pm 0.11$ (average reflectance), $0.09 \pm 0.09$ (parallel polarized light), and $0.11 \pm 0.21$ (birefringence). The Michelson contrasts for the depolarized light images were significantly higher than for the other three image types $(P<.0001$, for all tests, paired $t$ test). 


\section{DISCUSSION}

IN THE CURRENT STUDY, WE EVALUATED POLARIZATION analysis as a noninvasive technique for the evaluation of the extent of subretinal fluid and the localization of the pinpoint areas of light scattering in CSR. Polarization analysis could selectively emphasize the different features of the retina, visualizing more as opposed to less superficial layers depending upon polarization content. In the depolarized light image, the leakage areas presented as high intensity areas, indicating a high degree of multiple scattering of the returning light at those locations, in turn implying local tissue disorder. In recent OCT studies, various morphologic changes at the leakage areas were reported, for example, pigment epithelial detachment, 10 abridging lesion, 11 and bulges from retinal pigment epithelium. ${ }^{13}$ Our findings in the depolarized light image represent the morphologic changes at the leakage areas and agree with these OCT findings. In the chronic cases, CSR may be accompanied by retinal pigment epithelium atrophy, 14 extensive pigmentary change, ${ }^{14}$ or fibrinous exudates. ${ }^{1,15}$ Multiple dots and high intensity area around the leakage area in the depolarized light images could provide important information about the retinal pigment epithelium impairment around leakage areas.

The polarimetry technique has the advantage of being a noninvasive technique, which does not require pupil dilation, and provides en face results for a 15 degree region in one measurement. Testing time is short ( 0.7 seconds), and there is live viewing of the fundus during image acquisition to help ensure positive findings. The leakage point could readily be detected in the depolarized images without the need of repeated scans, so that existing treatment modalities could be applied with familiar landmarks. In some cases, the exact area for focal photocoagulation could not be determined, but an approximate area for photocoagulation might be obtained. Treatment outcomes based on the existing diagnostic and treatment modalities would then provide the necessary data as to the success of localization by polarimetry. Polarimetric analysis could also be a useful tool as a preparatory test before fluorescein angiography.

The average reflectance and the parallel polarized images did not show the leakage areas as well as the depolarized light image, but did localize in high contrast the neurosensory retinal detachment. This was expected since the former two image types are generated by polarization preserving light, emphasizing more superficial structures. The average reflectance image is similar to an image of a confocal scanning laser ophthalmoscope, which has been shown to emphasize more superficial retinal structures. ${ }^{16-18}$ The contrast of the fluid surrounding the leakage areas in CSR is high in these image types because there is a reduction of transmission of light both into the and out of the fluid, accompanied by a loss of polarization of the light that does pass through. Additionally, the average reflectance and parallel images have strong light return off the healthier regions of the retina, so that the resulting contrast with the darker region of fluid is high.

The birefringence image, which is generated from the modulation of the polarization preserving light, was not expected to enhance the visualization of key features of CSR. In 29 eyes of our case series, there was a macular bow-tie, which is attributable to the interaction of corneal and retinal birefringence. The presence of a macular bow-tie implies incomplete correction for corneal birefringence by the fixed compensator, ${ }^{19}$ yet the presence of a macular bow-tie could help to localize the fovea. In CSR cases, the foveal reflex can be missing because of abnormal light-tissue interactions. Localization of the fovea can be useful when focal photocoagulation is being considered. Even in eyes with sufficient compensation for the corneal birefringence and absence of a macular bow-tie, this structure might easily be regenerated by tilting of the patient's head. 
Polarimetry analysis has potentialities for further progress. Summary statistics from features or entire images may provide additional information, analogous to that used in nerve fiber layer analysis in glaucoma. The possible improvement of using full Mueller matrix polarimetry remains to be investigated. 20,21 These newer methods might more accurately separate polarization preserving from depolarized light, allowing more accurate quantification of retinal birefringence 22 as well as avoiding the possible artifacts of over-estimating depolarized light. ${ }^{7}$ Further study is necessary for the rapid, noninvasive assessment of the intrinsic changes in CSR.

\section{References}

1. Gass JDM. Stereoscopic atlas of macular disease, 3rd ed. St.Louis, Washington, DC, Toronto: Mosby, 1987:46 -59.

2. von Ruckmann A, Fitzke FW, Fan J, Halfyard A, Bird AC. Abnormalities of fundus autofluorescence in central serous retinopathy. Am J Ophthalmol 2002;133:780 -786. [PubMed: 12036669]

3. Yannuzzi LA, Rohrer KT, Tindel LJ, et al. Fluorescein angiography complication survey. Ophthalmology 1986;93:611- 617. [PubMed: 3523356]

4. Dreher AW, Reiter K, Weinreb RN. Spatially resolved birefringence of the retinal nerve fiber layer assessed with aretinal laser ellipsometry. Applied Optics 1992;31:3730 -3735.

5. Elsner AE, Miura M, Stewart JB, Kairala MB, Burns SA. Novel algorithms for polarization imaging resulting in improved quantification of retinal blood vessels. Stud Health Technol Inform 2003;94:59 -61. [PubMed: 15455864]

6. Burns SA, Elsner AE, Mellem-Kairala MB, Simmons RB. Improved contrast of subretinal structures using polarization analysis. Invest Ophthalmol Vis Sci 2003;44:4061- 4068. [PubMed: 12939329]

7. Mellem-Kairala MB, Elsner AE, Weber A, Simmons RB, Burns SA. Improved contrast of peripapillary hyperpigmentation using polarization analysis. Invest Ophthalmol Vis Sci 2005;46:1099-1106. [PubMed: 15728571]

8. Weber A, Cheney MC, Smithwick QYJ, Elsner AE. Polarimetric imaging and blood vessel quantification. Optics Express 2004;12:5178 -5190.

9. Demos SG, Alfano RR. Optical polarization imaging. Appl Opt 1997;36:150 -155.

10. Hee MR, Puliafito CA, Wong C, et al. Optical coherence tomography of central serous chorioretinopathy. Am J Ophthalmol 1995;120:65-74. [PubMed: 7611331]

11. Iida T, Hagimura N, Sato T, Kishi S. Evaluation of central serous chorioretinopathy with optical coherence tomography. Am J Ophthalmol 2000;129:16 -20. [PubMed: 10653407]

12. Weinreb RN, Shakiba S, Zangwill L. Scanning laser polarimetry to measure the nerve fiber layer of normal and glaucomatous eyes. Am J Ophthalmol 1995;119:627- 636. [PubMed: 7733188]

13. Montero JA, Ruiz-Moreno JM. Optical coherence tomography characterization of idiopathic central serous chorioretinopathy. Br J Ophthalmol 2005;89:562-564. [PubMed: 15834085]

14. Levine R, Brucker A, Robinson F. Long-term follow-up of idiopathic central serous chorioretinopathy by fluorescein angiography. Ophthalmology 1989;96:854 -859. [PubMed: 2740080]

15. Schatz H, McDonald H, Johnson R, et al. Subretinal fibrosis in central serous chorioretinopathy. Ophthalmology 1995;102:1077-1088. [PubMed: 9121756]

16. Miura M, Elsner AE, Beausencourt E, et al. Grading of infrared confocal scanning laser tomography and video displays of digitized color slides in exudative age-related macular degeneration. Retina 2002;22:300 -308. [PubMed: 12055463]

17. Miura M, Elsner AE, Osako M, et al. Spectral imaging of the area of internal limiting membrane peeling. Retina. Forthcoming.

18. Remky A, Arend O, Toonen F. Infrared imaging of central serous chorioretinopathy: A follow-up study. Acta Ophthalmol Scand 1998;76:339 -342. [PubMed: 9686849]

19. Knighton RW, Huang XR, Greenfield DS. Analytical model of scanning laser polarimetry for retinal nerve fiber layer assessment. Invest Ophthalmol Vis Sci 2002;43:383-392. [PubMed: 11818381]

20. Chipman RA. Polarimetry. In: Bass M, editor. Hand book of optics, 2nd edition. Volume 2. New York: McGraw-Hill,1995:1-28. 
21. Bueno JM. Measurement of parameters of polarization in the living human eye using imaging polarimetry. Vision Res 2000;40:3791-3799. [PubMed: 11090671]

22. Zhou Q, Weinreb RN. Individualized compensation of anterior segment birefringence during scanning laser polarimetry. Invest Ophthalmol Vis Sci 2002;43:2221-2228. [PubMed: 12091420]

\section{Biography}

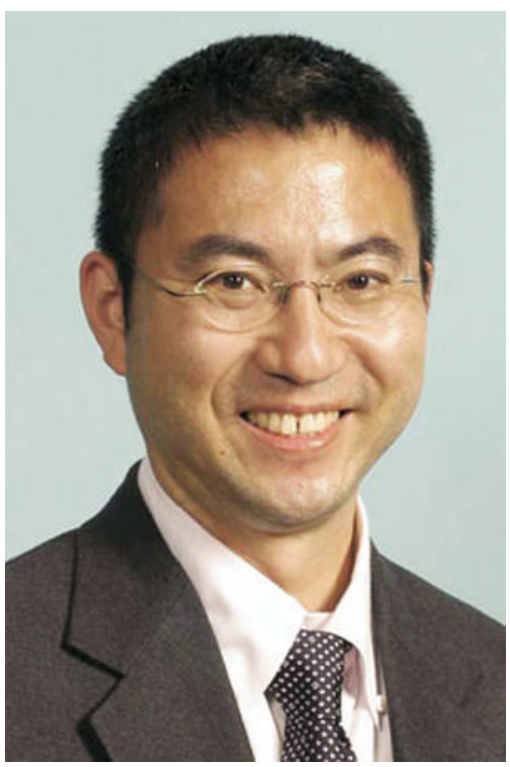

Miura, MD, graduated from Nippon Medical School. He was a Post-doctoral Fellow at the Schepens Eye Research Institute in Boston, Massachusetts. He is an Assistant Professor of Ophthalmology at Tokyo Medical University. His research interests include retinal imaging and macular disease. 


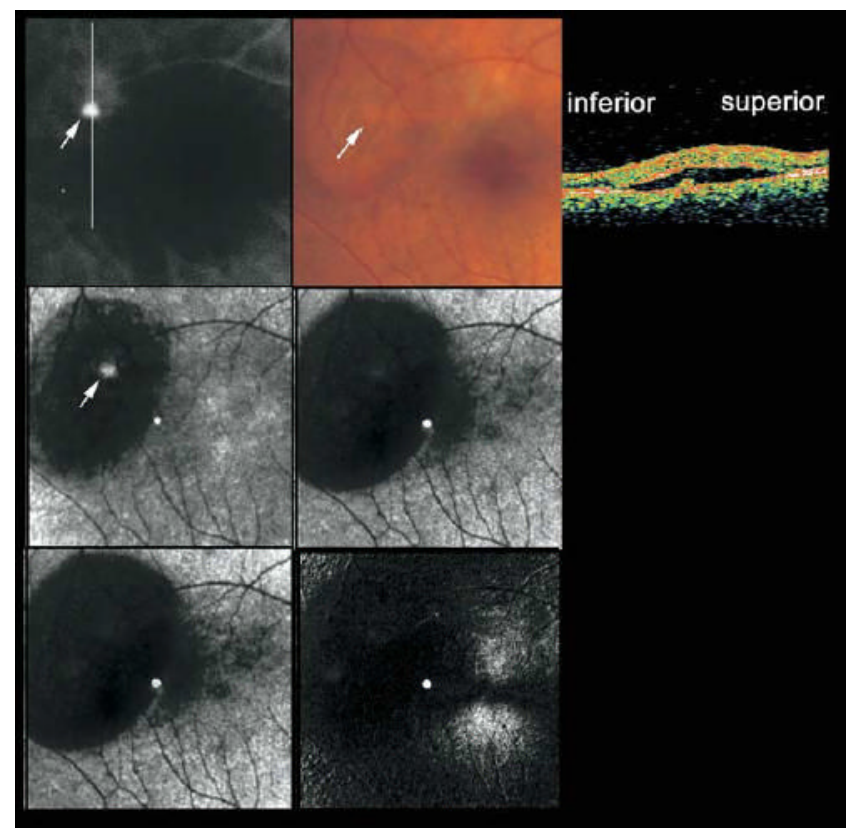

FIGURE 1.

Right eye (OD) of a 35-year-old Japanese man with central serous chorioretinopathy, reporting a paracentral relative scotoma of 2 weeks duration. Best-corrected visual acuity was 20/16 in both eyes. Fluorescein angiogram (top row, left) demonstrating dye leakage (white arrow) temporal to the macula. The white line indicates the scanning line for optical coherence tomography. Color fundus photograph (top row, center) showing a serous retinal detachment in the area temporal to the macula. The leakage areas showed faint grayishwhite regions (white arrow) in the color fundus photograph. Optical coherence tomography (top row, right), showing serous retinal detachment and a small retinal pigment epithelial detachment at the leakage area. A depolarized light image (middle row, left) clearly shows the leakage area as a bright dot (white arrow). The large, dark area surrounding the leakage point is consistent with the location of the serous retinal detachment. An average reflectance image (middle row, right) which shows the leakage area only faintly, but better shows the more superficial retinal features. A parallel polarized light image (bottom row, left) de-emphasizing the deeper features, such as the leakage point, and emphasizing the more superficial features, such as the serous retinal detachment. A birefringence image (bottom row, right) shows the typical macular bowtie, which pinpoints the location of the fovea, seemingly unaffected. The deeper features, such as leakage point, are not seen well in the birefringence image. The bright spot in the center of each polarimetry image is an artifact because of internal reflections in the GDx-N. 


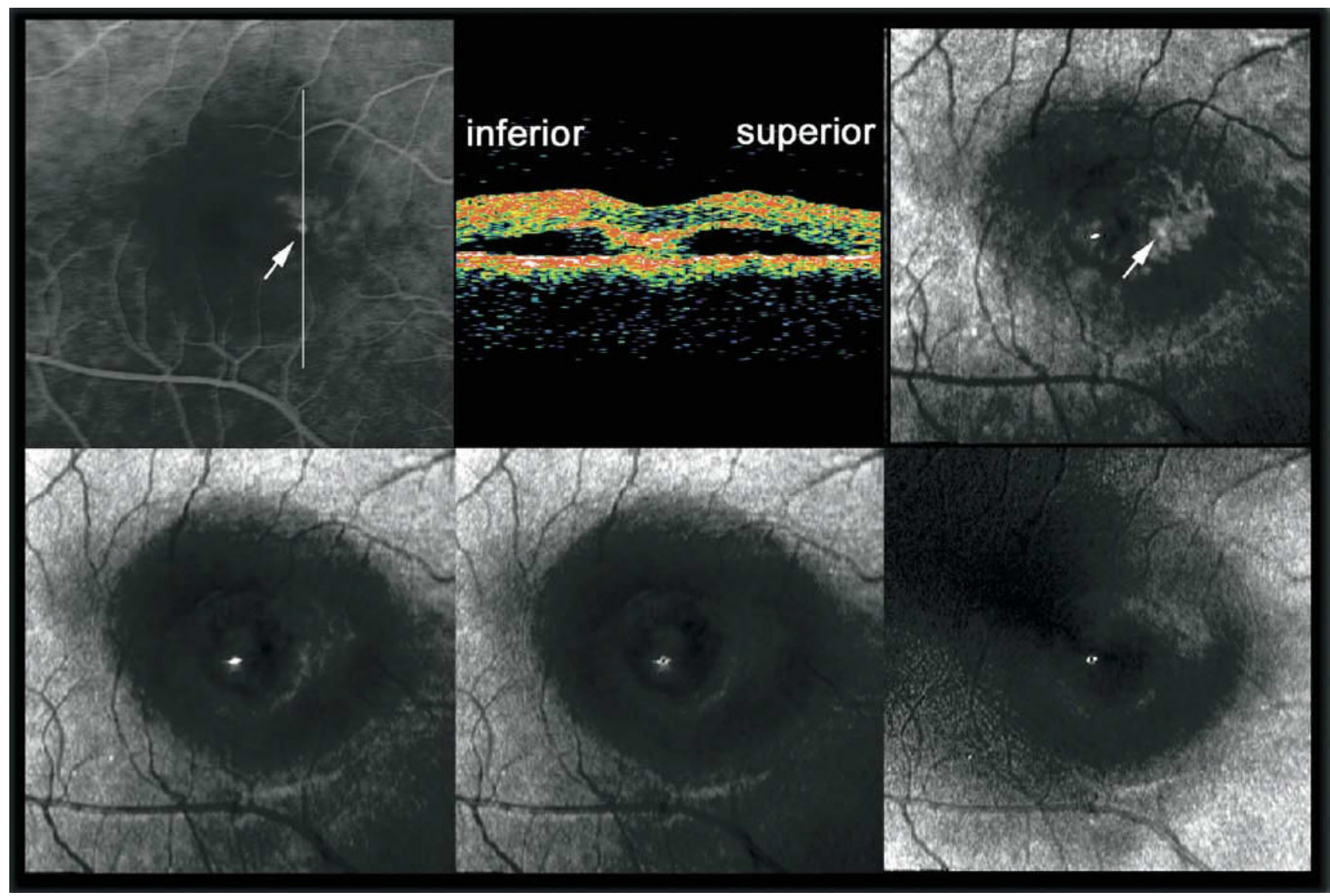

FIGURE 2.

Right eye (OD) of a 40-year-old Japanese man with central serous chorioretinopathy, reporting a central relative scotoma for 1 week. Best-corrected visual acuity in the right eye (OD) was 20/16 in both eyes. Fluorescein angiogram (top left) demonstrating dye leakage (white arrow) nasal to the macula. The white line indicates the scanning line for optical coherence tomography. Optical coherence tomography (top center) showing serous retinal detachment and a highly reflective lesion that bridged the detached neurosensory retina and the retinal pigment epithelium. A depolarized light image (top right) clearly showing the leakage area as a bright dot (white arrow), surrounded by a high intensity area. In the average reflectance image (bottom left), the parallel polarized light image (bottom center), or the birefringence image (bottom right), the leakage area could hardly be detected. The large dark area consistent with the location of the serous retinal detachment could be clearly visualized in a parallel polarized image and an average reflectance image. The bright spot on the center of the polarimetry images was an instrument artifact. 


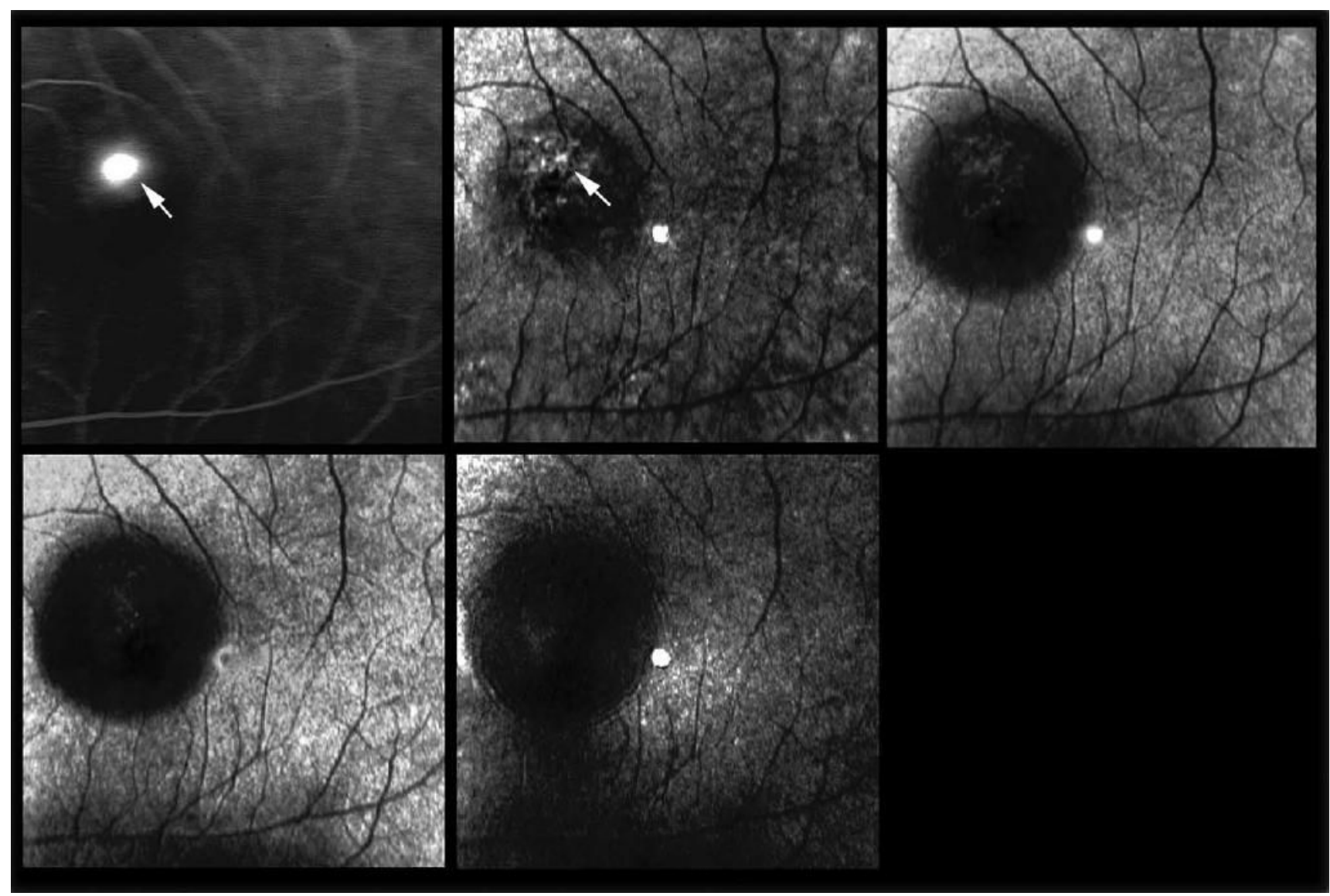

FIGURE 3.

Left eye (OS) of a 53-year-old Japanese man with central serous chorioretinopathy, reporting a blurring of central vision for 6 weeks. Best-corrected visual acuity was 20/16 in the right eye (OD) and 20/32 in the left eye (OS). Fluorescein angiogram (top left) demonstrating dye leakage (white arrow) near the macula. A depolarized light image (top center) clearly showing the leakage point as a bright dot (white arrow) accompanied by multiple bright dots. In the average reflectance image (top right), the parallel polarized light image (bottom left), or birefringence image (bottom right), the leakage area could be hardly detected. The neurosensory detachment was well-localized in the parallel polarized light image and average reflectance image. The birefringence image showing the macular bow-tie. A bright spot in the center of the polarimetry images was an instrument artifact. 


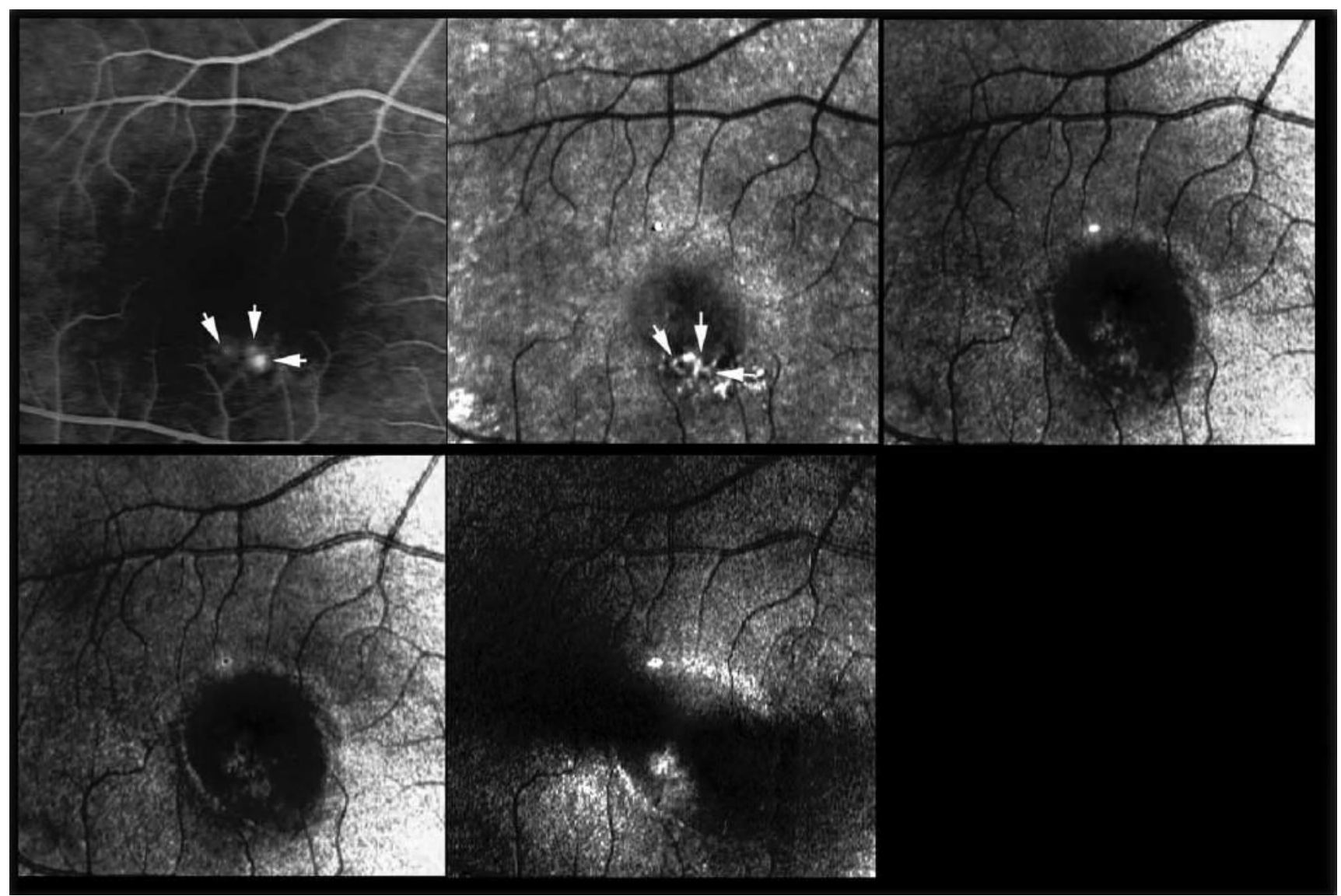

FIGURE 4.

Right eye (OD) of a 38-year-old Japanese woman with central serous chorioretinopathy, reporting metamorphopsia for 1 week. Best-corrected visual acuity was 20/32 in the right eye (OD) and 20/16 in the left eye (OS). Fluorescein angiogram (top left) demonstrating dye leakage (white arrows) inferior nasal to the macula. The depolarized light image (top center) clearly shows the leakage points as bright dots (white arrows) accompanied by small bright dots. In the average reflectance image (top right), the parallel polarized light image (bottom left), or the birefringence image (bottom right), leakage area could hardly be detected. The birefringence image shows the typical macular bow-tie. The bright spot in the center of the polarimetry images was an instrument artifact. 